\title{
Unitary equivalence classes of one-dimensional quantum walks II
}

\author{
Hiromichi Ohno ${ }^{1}$ (i)
}

Received: 30 April 2017 / Accepted: 11 October 2017 / Published online: 24 October 2017

C The Author(s) 2017. This article is an open access publication

\begin{abstract}
This study investigated the unitary equivalence classes of one-dimensional quantum walks with and without initial states. We determined the unitary equivalence classes of one-dimensional quantum walks, two-phase quantum walks with one defect, complete two-phase quantum walks, one-dimensional quantum walks with one defect and translation-invariant one-dimensional quantum walks.
\end{abstract}

Keywords Quantum walk · One-dimensional quantum walk · Unitary equivalence · Two-phase quantum walk with one defect - Complete two-phase quantum walk . One-dimensional quantum walks with one defect

\section{Introduction}

Quantum walks can be considered as a quantum analog of classical random walks and have been studied in various fields, such as quantum information theory and quantum probability theory. A quantum walk is defined by a pair $\left(U,\left\{\mathcal{H}_{v}\right\}_{v \in V}\right)$, in which $V$ is a countable set, $\left\{\mathcal{H}_{v}\right\}_{v \in V}$ is a family of separable Hilbert spaces, and $U$ is a unitary operator on $\mathcal{H}=\bigoplus_{v \in V} \mathcal{H}_{v}$ [20]. In this paper, we discuss one-dimensional (twostate) quantum walks, in which $V=\mathbb{Z}$ and $\mathcal{H}_{v}=\mathbb{C}^{2}$. These have been the subject of many previous studies [1-19,21-23].

It is important to clarify when we think of two quantum walks as being the same. We consider unitary equivalence of quantum walks in the sense of $[19,20]$. If two quantum walks are unitarily equivalent, then their digraphs and dimensions of their

\footnotetext{
$凶$ Hiromichi Ohno

h_ohno@shinshu-u.ac.jp

1 Department of Mathematics, Faculty of Engineering, Shinshu University, 4-17-1 Wakasato, Nagano 380-8553, Japan
} 
Hilbert spaces are the same. Furthermore, the probability distributions of the quantum walks are also the same. Consequently, we can think of unitarily equivalent quantum walks as being the same.

The aim of this paper is to determine the unitary equivalence classes of several types of one-dimensional quantum walks. This will enable us to grasp the whole image of such quantum walks. There are many results about explicit models of one-dimensional quantum walk [2,6-11,15-18,21,23]. The parameterization of unitary equivalence classes tells us what part of one-dimensional quantum walks were already investigated and what part should be studied. Moreover, some previous works researched onedimensional quantum walks using parameters [3-5]. The results in this paper reduce the parameters and make it easy to analyze them.

In the previous paper [19], we discussed some general properties of unitarily equivalent quantum walks. In particular, we proved that every one-dimensional quantum walk is the unitarily equivalent of one of the Ambainis types. We also presented the necessary and sufficient condition for defining a one-dimensional quantum walk as a Szegedy walk.

Unitary equivalence classes of simple quantum walks have been shown to be parameterized by a single parameter [12]. In contrast, there are several types of one-dimensional quantum walks, including two-phase quantum walks with one defect [6,7], complete two-phase quantum walks [11] and one-dimensional quantum walks with one defect $[4,8-10,17,18,23]$. A one-dimensional quantum walk with one defect is known for exhibiting localization. Two-phase quantum walks are considered as mathematical models of the topological insulator. In this study, we clarified the unitary equivalence classes of general one-dimensional quantum walks and of the above types of one-dimensional quantum walk, but excluding certain special cases.

When we analyze properties of an evolution operator $U$ such as the spectrum, eigenvalues and eigenvectors, it is not necessary to take into account an initial state. On the other hand, when studying the probability distribution of a quantum walk, an initial state must be set. Hence, we consider quantum walks both with and without initial states.

In Sect. 2, we investigate one-dimensional quantum walks without initial states and present our results: Two-phase quantum walks with one defect are parameterized by six parameters complete two-phase quantum walks by four parameters, and onedimensional quantum walks with one defect by four parameters. In Sect. 3, we show unitary equivalence classes of all the above types of one-dimensional quantum walk with an initial state.

\section{Unitary equivalence classes of one-dimensional quantum walks}

In this section, we investigate the unitary equivalence classes of several types of onedimensional quantum walks without initial states. We first consider the definition of a one-dimensional quantum walk and the unitary equivalence of such walks (see [19]).

Definition 1 Let $\mathcal{H}_{n}=\mathbb{C}^{2}$ for $n \in \mathbb{Z}$. A unitary operator $U$ on $\mathcal{H}=\bigoplus_{n \in \mathbb{Z}} \mathcal{H}_{n}$ is called a one-dimensional quantum walk if 


$$
\operatorname{rank} P_{n} U P_{m}= \begin{cases}1 & (m=n \pm 1) \\ 0 & (m \neq n \pm 1)\end{cases}
$$

for all $m, n \in \mathbb{Z}$, where $P_{n}$ is the projection onto $\mathcal{H}_{n}$.

$\mathrm{A}$ (pure) quantum state is represented by a unit vector in a Hilbert space. For $\lambda \in \mathbb{R}$, quantum states $\xi$ and $e^{\mathrm{i} \lambda} \xi$ in $\mathcal{H}$ are identified. Hence, the one-dimensional quantum walks $U$ and $e^{\mathrm{i} \lambda} U$ are also identified.

Definition 2 One-dimensional quantum walks $U_{1}$ and $U_{2}$ are unitarily equivalent if there exists a unitary $W=\bigoplus_{n \in \mathbb{Z}} W_{n}$ on $\mathcal{H}=\bigoplus_{n \in \mathbb{Z}} \mathcal{H}_{n}$ such that

$$
W U_{1} W^{*}=U_{2}
$$

Theorem 1 in [19] (see also the first paragraph of Section 5 in [19]) yields the next theorem.

Theorem 1 A one-dimensional quantum walk $U$ is described as follows:

$$
U=\sum_{n \in \mathbb{Z}}\left|\xi_{n-1, n}\right\rangle\left\langle\zeta_{n-1, n}|+| \xi_{n+1, n}\right\rangle\left\langle\zeta_{n+1, n}\right|,
$$

where $\left\{\xi_{n, n+1}, \xi_{n+1, n}\right\}_{n \in \mathbb{Z}}$ and $\left\{\zeta_{n, n+1}, \zeta_{n+1, n}\right\}_{n \in \mathbb{Z}}$ are orthonormal bases of $\mathcal{H}=$ $\bigoplus_{n \in \mathbb{Z}} \mathcal{H}_{n}$ with $\xi_{n, n+1}, \zeta_{n+1, n} \in \mathcal{H}_{n}$ and $\xi_{n+1, n}, \zeta_{n, n+1} \in \mathcal{H}_{n+1}$.

The unitary equivalence of a one-dimensional quantum walk can then be analyzed as follows.

Step 1 Assume that $U$ is described as in (1), and define a unitary operator $W_{1}$ on $\mathcal{H}$ as

$$
W_{1}=\bigoplus_{n \in \mathbb{Z}}\left|e_{1}^{n}\right\rangle\left\langle\xi_{n, n+1}|+| e_{2}^{n}\right\rangle\left\langle\xi_{n, n-1}\right|,
$$

where $\left\{e_{1}^{n}, e_{2}^{n}\right\}$ is the standard basis of $\mathcal{H}_{n}=\mathbb{C}^{2}$. Then,

$$
\begin{aligned}
& W_{1} U W_{1}^{*} \\
& =\sum_{n \in \mathbb{Z}}\left|W_{1} \xi_{n-1, n}\right\rangle\left\langle W_{1} \zeta_{n-1, n}|+| W_{1} \xi_{n+1, n}\right\rangle\left\langle W_{1} \zeta_{n+1, n}\right| \\
& =\sum_{n \in \mathbb{Z}}\left|e_{1}^{n-1}\right\rangle\left\langle e^{\mathrm{i} a_{n}} r_{n} e_{1}^{n}+e^{\mathrm{i} b_{n}} s_{n} e_{2}^{n}|+| e_{2}^{n+1}\right\rangle\left\langle e^{\mathrm{i} c_{n}} s_{n} e_{1}^{n}+e^{\mathrm{i} d_{n}} r_{n} e_{2}^{n}\right|
\end{aligned}
$$

for some $0 \leq r_{n} \leq 1$ and $a_{n}, b_{n}, c_{n}, d_{n} \in \mathbb{R}$ with $s_{n}=\sqrt{1-r_{n}^{2}}$ and

$$
a_{n}-b_{n}=c_{n}-d_{n}+\pi \quad(\bmod 2 \pi) .
$$

If there is no confusion, $(\bmod 2 \pi)$ can be omitted hereafter. 
Step 2 Define $g_{n} \in \mathbb{R}$ by $g_{0}=0$ and

$$
g_{n-1}-g_{n}=a_{n},
$$

inductively. Similarly, we define $h_{n} \in \mathbb{R}$ by $h_{0}=g_{-1}-b_{0}$ and

$$
h_{n+1}-h_{n}=d_{n},
$$

inductively. Then, by (3),

$$
\begin{aligned}
c_{n}-h_{n+1}+g_{n} & =c_{n}-h_{n}-d_{n}+g_{n-1}-a_{n} \\
& =-\left(b_{n}-g_{n-1}+h_{n}\right)+\pi \quad(\bmod 2 \pi) .
\end{aligned}
$$

Let $W_{2}$ be a unitary operator defined by

$$
W_{2}=\bigoplus_{n \in \mathbb{Z}} e^{\mathrm{i} g_{n}}\left|e_{1}^{n}\right\rangle\left\langle e_{1}^{n}\left|+e^{\mathrm{i} h_{n}}\right| e_{2}^{n}\right\rangle\left\langle e_{2}^{n}\right|
$$

By definitions and (4),

$$
\begin{aligned}
W_{2} & W_{1} U W_{1}^{*} W_{2}^{*} \\
= & \sum_{n \in \mathbb{Z}}\left|e_{1}^{n-1}\right\rangle\left\langle e^{\mathrm{i}\left(a_{n}-g_{n-1}+g_{n}\right)} r_{n} e_{1}^{n}+e^{\mathrm{i}\left(b_{n}-g_{n-1}+h_{n}\right)} s_{n} e_{2}^{n}\right| \\
& +\left|e_{2}^{n+1}\right\rangle\left\langle e^{\mathrm{i}\left(c_{n}-h_{n+1}+g_{n}\right)} s_{n} e_{1}^{n}+e^{\mathrm{i}\left(d_{n}-h_{n+1}+h_{n}\right)} r_{n} e_{2}^{n}\right| \\
= & \sum_{n \in \mathbb{Z}}\left|e_{1}^{n-1}\right\rangle\left\langle r_{n} e_{1}^{n}+e^{\mathrm{i} k_{n}} s_{n} e_{2}^{n}|+| e_{2}^{n+1}\right\rangle\left\langle-e^{-\mathrm{i} k_{n}} s_{n} e_{1}^{n}+r_{n} e_{2}^{n}\right|,
\end{aligned}
$$

where $k_{n}=b_{n}-g_{n-1}+h_{n}$. Here,

$$
k_{0}=b_{0}-g_{-1}+h_{0}=0
$$

Step 3 Let $\ell=k_{1} / 2, p_{n}=n k_{1} / 2$ and $q_{n}=-n k_{1} / 2$, and let

$$
W_{3}=\bigoplus_{n \in \mathbb{Z}} e^{\mathrm{i} p_{n}}\left|e_{1}^{n}\right\rangle\left\langle e_{1}^{n}\left|+e^{\mathrm{i} q_{n}}\right| e_{2}^{n}\right\rangle\left\langle e_{2}^{n}\right|
$$


Then,

$$
\begin{aligned}
e^{\mathrm{i} \ell} & W_{3} W_{2} W_{1} U W_{1}^{*} W_{2}^{*} W_{3}^{*} \\
= & \sum_{n \in \mathbb{Z}}\left|e_{1}^{n-1}\right\rangle\left\langle e^{\mathrm{i}\left(-p_{n-1}+p_{n}-\ell\right)} r_{n} e_{1}^{n}+e^{\mathrm{i}\left(k_{n}-p_{n-1}+q_{n}-\ell\right)} s_{n} e_{2}^{n}\right| \\
& +\left|e_{2}^{n+1}\right\rangle\left\langle-e^{\mathrm{i}\left(-k_{n}-q_{n+1}+p_{n}-\ell\right)} s_{n} e_{1}^{n}+e^{\mathrm{i}\left(-q_{n+1}+q_{n}-\ell\right)} r_{n} e_{2}^{n}\right| \\
= & \sum_{n \in \mathbb{Z}}\left|e_{1}^{n-1}\right\rangle\left\langle r_{n} e_{1}^{n}+e^{\mathrm{i}\left(k_{n}-n k_{1}\right)} s_{n} e_{2}^{n}|+| e_{2}^{n+1}\right\rangle\left\langle-e^{\mathrm{i}\left(-k_{n}+n k_{1}\right)} s_{n} e_{1}^{n}+r_{n} e_{2}^{n}\right| \\
= & \sum_{n \in \mathbb{Z}}\left|e_{1}^{n-1}\right\rangle\left\langle r_{n} e_{1}^{n}+e^{\mathrm{i} \theta_{n}} s_{n} e_{2}^{n}|+| e_{2}^{n+1}\right\rangle\left\langle-e^{-\mathrm{i} \theta_{n}} s_{n} e_{1}^{n}+r_{n} e_{2}^{n}\right|,
\end{aligned}
$$

where $\theta_{n}=k_{n}-n k_{1}$. Here, $\theta_{0}=\theta_{1}=0$.

Now, we are ready to prove the next theorem.

Theorem 2 A one-dimensional quantum walk $U$ is unitarily equivalent to

$$
U_{r, \theta}=\sum_{n \in \mathbb{Z}}\left|e_{1}^{n-1}\right\rangle\left\langle r_{n} e_{1}^{n}+e^{\mathrm{i} \theta_{n}} s_{n} e_{2}^{n}|+| e_{2}^{n+1}\right\rangle\left\langle-e^{-\mathrm{i} \theta_{n}} s_{n} e_{1}^{n}+r_{n} e_{2}^{n}\right|
$$

for some $0 \leq r_{n} \leq 1$ and $\theta_{n} \in \mathbb{R}$ with $s_{n}=\sqrt{1-r_{n}^{2}}$ and $\theta_{0}=\theta_{1}=0$. Moreover, when $0<r_{n}, r_{n}^{\prime}<1$ and $\theta_{n}, \theta_{n}^{\prime} \in[0,2 \pi), U_{r, \theta}$ and $U_{r^{\prime}, \theta^{\prime}}$ are unitarily equivalent if and only if $r=r^{\prime}$ and $\theta=\theta^{\prime}$.

Proof We have already proved the first part of this theorem.

We assume that $U_{r, \theta}$ and $U_{r^{\prime}, \theta^{\prime}}$ are unitarily equivalent, where $0<r_{n}, r_{n}^{\prime}<1$ and $\theta_{n}, \theta_{n}^{\prime} \in[0,2 \pi)$ with $\theta_{0}=\theta_{1}=\theta_{0}^{\prime}=\theta_{1}^{\prime}=0$. Then, there exist $\lambda \in \mathbb{R}$ and a unitary operator $W=\bigoplus_{n \in \mathbb{Z}} W_{n}$ on $\mathcal{H}=\bigoplus_{n \in \mathbb{Z}} \mathcal{H}_{n}$ such that

$$
e^{\mathrm{i} \lambda} W U_{r, \theta} W^{*}=U_{r^{\prime}, \theta^{\prime}} .
$$

Then,

$$
\begin{aligned}
& e^{\mathrm{i} \lambda} W U_{r, \theta} W^{*} \\
& \quad=e^{\mathrm{i} \lambda} \sum_{n \in \mathbb{Z}}\left|W e_{1}^{n-1}\right\rangle\left\langle r_{n} W e_{1}^{n}+e^{\mathrm{i} \theta_{n}} s_{n} W e_{2}^{n}|+| W e_{2}^{n+1}\right\rangle\left\langle-e^{-\mathrm{i} \theta_{n}} s_{n} W e_{1}^{n}+r_{n} W e_{2}^{n}\right|
\end{aligned}
$$

and

$$
U_{r^{\prime}, \theta^{\prime}}=\sum_{n \in \mathbb{Z}}\left|e_{1}^{n-1}\right\rangle\left\langle r_{n}^{\prime} e_{1}^{n}+e^{\mathrm{i} \theta_{n}^{\prime}} s_{n}^{\prime} e_{2}^{n}|+| e_{2}^{n+1}\right\rangle\left\langle-e^{-\mathrm{i} \theta_{n}^{\prime}} s_{n}^{\prime} e_{1}^{n}+r_{n}^{\prime} e_{2}^{n}\right|
$$


Since $P_{n \pm 1} e^{\mathrm{i} \lambda} W U_{r, \theta} W^{*} P_{n}=P_{n \pm 1} U_{r^{\prime}, \theta^{\prime}} P_{n}$ for all $n \in \mathbb{Z}, W e_{1}^{n}$ and $W e_{2}^{n}$ are described as $W e_{1}^{n}=e^{\mathrm{i} u_{n}} e_{1}^{n}$ and $W e_{2}^{n}=e^{\mathrm{i} v_{n}} e_{2}^{n}$ for some $u_{n}, v_{n} \in \mathbb{R}$. Then,

$$
\begin{aligned}
& e^{\mathrm{i} \lambda} W U_{r, \theta} W^{*} \\
& =\sum_{n \in \mathbb{Z}}\left|e_{1}^{n-1}\right\rangle\left\langle e^{\mathrm{i}\left(-u_{n-1}+u_{n}-\lambda\right)} r_{n} e_{1}^{n}+e^{\mathrm{i}\left(\theta_{n}-u_{n-1}+v_{n}-\lambda\right)} s_{n} e_{2}^{n}\right| \\
& \quad+\left|e_{2}^{n+1}\right\rangle\left\langle-e^{\mathrm{i}\left(-\theta_{n}-v_{n+1}+u_{n}-\lambda\right)} s_{n} e_{1}^{n}+e^{\mathrm{i}\left(-v_{n+1}+v_{n}-\lambda\right)} r_{n} e_{2}^{n}\right| .
\end{aligned}
$$

Comparing the coefficients of $\left|e_{1}^{n-1}\right\rangle\left\langle e_{1}^{n}\right|$ and $\left|e_{2}^{n+1}\right\rangle\left\langle e_{2}^{n}\right|$ yields

$$
-u_{n-1}+u_{n}-\lambda=0, \quad-v_{n+1}+v_{n}-\lambda=0
$$

Here, we can assume that $u_{0}=0$ because $W U W^{*}=\left(e^{\mathrm{i} w} W\right) U\left(e^{\mathrm{i} w} W\right)^{*}$ for any $w \in \mathbb{R}$. Therefore, $u_{n}=n \lambda$. Moreover, the coefficients of $\left|e_{1}^{-1}\right\rangle\left\langle e_{2}^{0}\right|$ imply

$$
0=\theta_{0}^{\prime}=\theta_{0}-u_{-1}+v_{0}-\lambda
$$

with the result that $v_{0}=0$. Hence, $v_{n}=-n \lambda$. Similarly, the coefficients of $\left|e_{1}^{0}\right\rangle\left\langle e_{2}^{1}\right|$ imply

$$
0=\theta_{1}^{\prime}=\theta_{1}-u_{0}+v_{1}-\lambda=-2 \lambda(\bmod 2 \pi),
$$

with the result that $\lambda=0, \pi$. When $\lambda=0, u_{n}=v_{n}=0$, and therefore, $W=I_{\mathcal{H}}$ and $U_{r, \theta}=U_{r^{\prime}, \theta^{\prime}}$. When $\lambda=\pi, u_{n}=n \pi$ and $v_{n}=-n \pi=n \pi(\bmod 2 \pi)$. In this case, $W=\bigoplus_{n \in \mathbb{Z}}(-1)^{n} I_{\mathcal{H}_{n}}$. By (5), we have $e^{\mathrm{i} \lambda} W U_{r, \theta} W^{*}=U_{r, \theta}$, and therefore, $U_{r, \theta}=U_{r^{\prime}, \theta^{\prime}}$. It is easy to see that $U_{r, \theta}=U_{r^{\prime}, \theta^{\prime}}$ implies that $r=r^{\prime}$ and $\theta=\theta^{\prime}$.

The converse is obvious.

This theorem says that the unitary equivalence classes of general one-dimensional quantum walks are described by $\left\{U_{r, \theta}\right\}$ with $\theta_{0}=\theta_{1}=0$.

The operator $U_{r, \theta}$ is similar to the CMV matrix introduced in [3,5]. However, our approach and result are different in three ways from those used in [3,5]. First, our starting point is the general one-dimensional quantum walk. Second, we add the condition $\theta_{0}=\theta_{1}=0$. Third, with the exception of certain special cases, $U_{r, \theta}$ and $U_{r^{\prime}, \theta^{\prime}}$ are not unitarily equivalent if $r \neq r^{\prime}$ or $\theta \neq \theta^{\prime}$.

We next consider some special cases of the one-dimensional quantum walk and introduce four types of one-dimensional quantum walk.

Definition 3 Let $U$ be a one-dimensional quantum walk expressed by

$$
U=\sum_{n \in \mathbb{Z}}\left|\xi_{n-1, n}\right\rangle\left\langle\zeta_{n-1, n}|+| \xi_{n+1, n}\right\rangle\left\langle\zeta_{n+1, n}\right| .
$$

(i) $U$ is called a translation-invariant quantum walk if there exist vectors $\xi_{1}, \xi_{2}, \zeta_{1}, \zeta_{2} \in \mathbb{C}^{2}$ such that

$$
\xi_{n, n+1}=\xi_{1}, \quad \xi_{n, n-1}=\xi_{2}, \quad \zeta_{n-1, n}=\zeta_{1}, \quad \zeta_{n+1, n}=\zeta_{2}
$$


for all $n \in \mathbb{Z}$. In other words, the vectors $\xi_{n, n+1}$ are the same, and $\xi_{n, n-1}, \zeta_{n-1, n}$, $\zeta_{n+1, n}$ also satisfy similar conditions.

(ii) $U$ is called a one-dimensional quantum walk with one defect if there exist vectors $\xi_{1}, \xi_{2}, \zeta_{1}, \zeta_{2} \in \mathbb{C}^{2}$ such that

$$
\xi_{n, n+1}=\xi_{1}, \quad \xi_{n, n-1}=\xi_{2}, \quad \zeta_{n-1, n}=\zeta_{1}, \quad \zeta_{n+1, n}=\zeta_{2}
$$

for all $n \in \mathbb{Z} \backslash\{0\}$. In other words, the vectors $\xi_{n, n+1}$ are the same except $n \neq 0$, and $\xi_{n, n-1}, \zeta_{n-1, n}, \zeta_{n+1, n}$ also satisfy similar conditions.

(iii) $U$ is called a complete two-phase quantum walk if there exist vectors $\xi_{1}^{+}, \xi_{1}^{-}, \xi_{2}^{+}, \xi_{2}^{-}, \zeta_{1}^{+}, \zeta_{1}^{-}, \zeta_{2}^{+}, \zeta_{2}^{-} \in \mathbb{C}^{2}$ such that

$$
\xi_{n, n+1}=\xi_{1}^{+}, \quad \xi_{n, n-1}=\xi_{2}^{+}, \quad \zeta_{n-1, n}=\zeta_{1}^{+}, \quad \zeta_{n+1, n}=\zeta_{2}^{+}
$$

for all $n \geq 0$ and

$$
\xi_{n, n+1}=\xi_{1}^{-}, \quad \xi_{n, n-1}=\xi_{2}^{-}, \quad \zeta_{n-1, n}=\zeta_{1}^{-}, \quad \zeta_{n+1, n}=\zeta_{2}^{-}
$$

for all $n \leq-1$.

(iv) $U$ is called a two-phase quantum walk with one defect if there exist vectors $\xi_{1}^{+}, \xi_{1}^{-}, \xi_{2}^{+}, \xi_{2}^{-}, \zeta_{1}^{+}, \zeta_{1}^{-}, \zeta_{2}^{+}, \zeta_{2}^{-} \in \mathbb{C}^{2}$ such that

$$
\xi_{n, n+1}=\xi_{1}^{+}, \quad \xi_{n, n-1}=\xi_{2}^{+}, \quad \zeta_{n-1, n}=\zeta_{1}^{+}, \quad \zeta_{n+1, n}=\zeta_{2}^{+}
$$

for all $n \geq 1$ and

$$
\xi_{n, n+1}=\xi_{1}^{-}, \quad \xi_{n, n-1}=\xi_{2}^{-}, \quad \zeta_{n-1, n}=\zeta_{1}^{-}, \quad \zeta_{n+1, n}=\zeta_{2}^{-}
$$

for all $n \leq-1$

By definition, two-phase quantum walks with one defect include quantum walks of other three types. Hence, we first investigate the unitary equivalence classes of two-phase quantum walks with one defect.

Theorem 3 A two-phase quantum walk $U$ with one defect is unitarily equivalent to

$$
\begin{aligned}
U_{r_{+}, r_{-}, r_{0}, \mu_{1}, \mu_{2}, \mu_{3}}= & \left|e_{1}^{-1}\right\rangle\left\langle r_{0} e_{1}^{0}+e^{\mathrm{i} \mu_{1}} s_{0} e_{2}^{0}|+| e_{2}^{1}\right\rangle\left\langle-e^{\mathrm{i} \mu_{2}} s_{0} e_{1}^{0}+e^{\mathrm{i}\left(\mu_{1}+\mu_{2}\right)} r_{0} e_{2}^{0}\right| \\
& +\sum_{n \geq 1}\left|e_{1}^{n-1}\right\rangle\left\langle r_{+} e_{1}^{n}+s_{+} e_{2}^{n}|+| e_{2}^{n+1}\right\rangle\left\langle-e^{\mathrm{i} \mu_{3}} s_{+} e_{1}^{n}+e^{\mathrm{i} \mu_{3}} r_{+} e_{2}^{n}\right| \\
& +\sum_{n \leq-1}\left|e_{1}^{n-1}\right\rangle\left\langle r_{-} e_{1}^{n}+s_{-} e_{2}^{n}|+| e_{2}^{n+1}\right\rangle\left\langle-s_{-} e_{1}^{n}+r_{-} e_{2}^{n}\right|
\end{aligned}
$$

for some $0 \leq r_{+}, r_{-}, r_{0} \leq 1$ and $\mu_{1}, \mu_{2}, \mu_{3} \in \mathbb{R}$ with $s_{\varepsilon}=\sqrt{1-r_{\varepsilon}^{2}}(\varepsilon=+,-, 0)$. We write $U_{r_{+}, r_{-}, r_{0}, \mu_{1}, \mu_{2}, \mu_{3}}=U_{r, \mu}$ for short. Moreover, when $0<r_{\varepsilon}, r_{\varepsilon}^{\prime}<1(\varepsilon=$ $+,-, 0)$ and $\mu_{i}, \mu_{i}^{\prime} \in[0,2 \pi)(i=1,2,3), U_{r, \mu}$ and $U_{r^{\prime}, \mu^{\prime}}$ are unitarily equivalent if and only if $r=r^{\prime}, \mu=\mu^{\prime}$. 
Proof Let

$$
U=\sum_{n \in \mathbb{Z}}\left|\xi_{n-1, n}\right\rangle\left\langle\zeta_{n-1, n}|+| \xi_{n+1, n}\right\rangle\left\langle\zeta_{n+1, n}\right|
$$

be a two-phase quantum walk with one defect. Then, by (6) and (7), Eq. (2) can be written as

$$
\begin{aligned}
& W_{1} U W_{1}^{*} \\
& =\left|e_{1}^{-1}\right\rangle\left\langle e^{\mathrm{i} a_{0}} r_{0} e_{1}^{0}+e^{\mathrm{i} b_{0}} s_{0} e_{2}^{0}|+| e_{2}^{1}\right\rangle\left\langle e^{\mathrm{i} c_{0}} s_{0} e_{1}^{0}+e^{\mathrm{i} d_{0}} r_{0} e_{2}^{0}\right| \\
& \quad+\sum_{n \geq 1}\left|e_{1}^{n-1}\right\rangle\left\langle e^{\mathrm{i} a_{+}} r_{+} e_{1}^{n}+e^{\mathrm{i} b_{+}} s_{+} e_{2}^{n}|+| e_{2}^{n+1}\right\rangle\left\langle e^{\mathrm{i} c_{+}} s_{+} e_{1}^{n}+e^{\mathrm{i} d_{+}} r_{+} e_{2}^{n}\right| \\
& \quad+\sum_{n \leq-1}\left|e_{1}^{n-1}\right\rangle\left\langle e^{\mathrm{i} a_{-}} r_{-} e_{1}^{n}+e^{\mathrm{i} b_{-}} s_{-} e_{2}^{n}|+| e_{2}^{n+1}\right\rangle\left\langle e^{\mathrm{i} c_{-}} s_{-} e_{1}^{n}+e^{\mathrm{i} d_{-}} r_{-} e_{2}^{n}\right|
\end{aligned}
$$

for some $0 \leq r_{\varepsilon} \leq 1$ and $a_{\varepsilon}, b_{\varepsilon}, c_{\varepsilon}, d_{\varepsilon} \in \mathbb{R}$ with $s_{\varepsilon}=\sqrt{1-r_{\varepsilon}^{2}}(\varepsilon=+,-, 0)$.

We can modify Step 2 as follows:

Step $2^{\prime}$ Let $\ell=\left(b_{-}+c_{-}+\pi\right) / 2$. Define $g_{n}, h_{n} \in \mathbb{R}$ by

$$
g_{n}= \begin{cases}n\left(\ell-a_{+}\right) & (n \geq 0) \\ n\left(\ell-a_{-}\right)-a_{-}+a_{0} & (n \leq-1)\end{cases}
$$

and

$$
h_{n}=\left\{\begin{array}{ll}
(n-1)\left(\ell-a_{+}\right)-b_{+}+\ell & (n \geq 1) \\
(n-1)\left(\ell-a_{-}\right)+c_{-}+a_{0}-a_{-}-\ell+\pi & (n \leq 0)
\end{array},\right.
$$

and a unitary $W_{2}$ on $\mathcal{H}$ by

$$
W_{2}=\bigoplus_{n \in \mathbb{Z}} e^{\mathrm{i} g_{n}}\left|e_{1}^{n}\right\rangle\left\langle e_{1}^{n}\left|+e^{\mathrm{i} h_{n}}\right| e_{2}^{n}\right\rangle\left\langle e_{2}^{n}\right|
$$

Then, using $a_{\varepsilon}+d_{\varepsilon}+\pi=b_{\varepsilon}+c_{\varepsilon}$,

$$
\begin{aligned}
e^{\mathrm{i} \ell} & W_{2} W_{1} U W_{1}^{*} W_{2}^{*} \\
= & \left|e_{1}^{-1}\right\rangle\left\langle e^{\mathrm{i}\left(a_{0}-g_{-1}+g_{0}-\ell\right)} r_{0} e_{1}^{0}+e^{\mathrm{i}\left(b_{0}-g_{-1}+h_{0}-\ell\right)} s_{0} e_{2}^{0}\right| \\
& +\left|e_{2}^{1}\right\rangle\left\langle e^{\mathrm{i}\left(c_{0}-h_{1}+g_{0}-\ell\right)} s_{0} e_{1}^{0}+e^{\mathrm{i}\left(d_{0}-h_{1}+h_{0}-\ell\right)} r_{0} e_{2}^{0}\right| \\
& +\sum_{n \geq 1}\left|e_{1}^{n-1}\right\rangle\left\langle e^{\mathrm{i}\left(a_{+}-g_{n-1}+g_{n}-\ell\right)} r_{+} e_{1}^{n}+e^{\mathrm{i}\left(b_{+}-g_{n-1}+h_{n}-\ell\right)} s_{+} e_{2}^{n}\right| \\
& +\left|e_{2}^{n+1}\right\rangle\left\langle e^{\mathrm{i}\left(c_{+}-h_{n+1}+g_{n}-\ell\right)} s_{+} e_{1}^{n}+e^{\mathrm{i}\left(d_{+}-h_{n+1}+h_{n}-\ell\right)} r_{+} e_{2}^{n}\right| \\
& +\sum_{n \leq-1}\left|e_{1}^{n-1}\right\rangle\left\langle e^{\mathrm{i}\left(a_{-}-g_{n-1}+g_{n}-\ell\right)} r_{-} e_{1}^{n}+e^{\mathrm{i}\left(b_{-}-g_{n-1}+h_{n}-\ell\right)} s_{-} e_{2}^{n}\right| \\
& +\left|e_{2}^{n+1}\right\rangle\left\langle e^{\mathrm{i}\left(c_{-}-h_{n+1}+g_{n}-\ell\right)} s_{-} e_{1}^{n}+e^{\mathrm{i}\left(d_{-}-h_{n+1}+h_{n}-\ell\right)} r_{-} e_{2}^{n}\right|
\end{aligned}
$$




$$
\begin{aligned}
= & \left|e_{1}^{-1}\right\rangle\left\langle r_{0} e_{1}^{0}+e^{\mathrm{i}\left(b_{0}-b_{-}\right)} s_{0} e_{2}^{0}\right| \\
& +\left|e_{2}^{1}\right\rangle\left\langle-e^{\mathrm{i}\left(b_{+}-b_{-}+c_{0}-c_{-}\right)} s_{0} e_{1}^{0}+e^{\mathrm{i}\left(b_{0}+b_{+}-2 b_{-}+c_{0}-c_{-}\right)} r_{0} e_{2}^{0}\right| \\
& +\sum_{n \geq 1}\left|e_{1}^{n-1}\right\rangle\left\langle r_{+} e_{1}^{n}+s_{+} e_{2}^{n}\right| \\
& +\left|e_{2}^{n+1}\right\rangle\left\langle-e^{\mathrm{i}\left(b_{+}-b_{-}+c_{+}-c_{-}\right)} s_{+} e_{1}^{n}+e^{\mathrm{i}\left(b_{+}-b_{-}+c_{+}-c_{-}\right)} r_{+} e_{2}^{n}\right| \\
& +\sum_{n \leq-1}\left|e_{1}^{n-1}\right\rangle\left\langle r_{-} e_{1}^{n}+s_{-} e_{2}^{n}|+| e_{2}^{n+1}\right\rangle\left\langle-s_{-} e_{1}^{n}+r_{-} e_{2}^{n}\right| \\
= & \left|e_{1}^{-1}\right\rangle\left\langle r_{0} e_{1}^{0}+e^{\mathrm{i} \mu_{1}} s_{0} e_{2}^{0}|+| e_{2}^{1}\right\rangle\left\langle-e^{\mathrm{i} \mu_{2}} s_{0} e_{1}^{0}+e^{\mathrm{i}\left(\mu_{1}+\mu_{2}\right)} r_{0} e_{2}^{0}\right| \\
& +\sum_{n \geq 1}\left|e_{1}^{n-1}\right\rangle\left\langle r_{+} e_{1}^{n}+s_{+} e_{2}^{n}|+| e_{2}^{n+1}\right\rangle\left\langle-e^{\mathrm{i} \mu_{3}} s_{+} e_{1}^{n}+e^{\mathrm{i} \mu_{3}} r_{+} e_{2}^{n}\right| \\
& +\sum_{n \leq-1}\left|e_{1}^{n-1}\right\rangle\left\langle r_{-} e_{1}^{n}+s_{-} e_{2}^{n}|+| e_{2}^{n+1}\right\rangle\left\langle-s_{-} e_{1}^{n}+r_{-} e_{2}^{n}\right| \\
= & U_{r, \mu},
\end{aligned}
$$

where $\mu_{1}=b_{0}-b_{-}, \mu_{2}=b_{+}-b_{-}+c_{0}-c_{-}$and $\mu_{3}=b_{+}-b_{-}+c_{+}-c_{-}$. This shows the first assertion of this theorem.

Next, we assume that $U_{r, \mu}$ and $U_{r^{\prime}, \mu^{\prime}}$ are unitarily equivalent, where $0<r_{\varepsilon}, r_{\varepsilon}^{\prime}<1$ and $\mu_{i}, \mu_{i}^{\prime} \in[0,2 \pi)$. Then, there exist $\lambda \in \mathbb{R}$ and a unitary operator $W=\bigoplus_{n \in \mathbb{Z}} W_{n}$ on $\mathcal{H}=\bigoplus_{n \in \mathbb{Z}} \mathcal{H}_{n}$ such that

$$
e^{\mathrm{i} \lambda} W U_{r, \mu} W^{*}=U_{r^{\prime}, \mu^{\prime}} .
$$

Here,

$$
\begin{aligned}
& e^{\mathrm{i} \lambda} W U_{r, \mu} W^{*} \\
& =e^{\mathrm{i} \lambda}\left|W e_{1}^{-1}\right\rangle\left\langle r_{0} W e_{1}^{0}+e^{\mathrm{i} \mu_{1}} s_{0} W e_{2}^{0}\left|+e^{\mathrm{i} \lambda}\right| W e_{2}^{1}\right\rangle\left\langle-e^{\mathrm{i} \mu_{2}} s_{0} W e_{1}^{0}+e^{\mathrm{i}\left(\mu_{1}+\mu_{2}\right)} r_{0} W e_{2}^{0}\right| \\
& \quad+e^{\mathrm{i} \lambda} \sum_{n \geq 1}\left|W e_{1}^{n-1}\right\rangle\left\langle r_{+} W e_{1}^{n}+s_{+} W e_{2}^{n}|+| W e_{2}^{n+1}\right\rangle\left\langle-e^{\mathrm{i} \mu_{3}} s_{+} W e_{1}^{n}+e^{\mathrm{i} \mu_{3}} r_{+} W e_{2}^{n}\right| \\
& \quad+e^{\mathrm{i} \lambda} \sum_{n \leq-1}\left|W e_{1}^{n-1}\right\rangle\left\langle r_{-} W e_{1}^{n}+s_{-} W e_{2}^{n}|+| W e_{2}^{n+1}\right\rangle\left\langle-s_{-} W e_{1}^{n}+r_{-} W e_{2}^{n}\right|
\end{aligned}
$$

and

$$
\begin{aligned}
U_{r^{\prime}, \mu^{\prime}}= & \left|e_{1}^{-1}\right\rangle\left\langle r_{0}^{\prime} e_{1}^{0}+e^{\mathrm{i} \mu_{1}^{\prime}} s_{0}^{\prime} e_{2}^{0}|+| e_{2}^{1}\right\rangle\left\langle-e^{\mathrm{i} \mu_{2}^{\prime}} s_{0}^{\prime} e_{1}^{0}+e^{\mathrm{i}\left(\mu_{1}^{\prime}+\mu_{2}^{\prime}\right)} r_{0}^{\prime} e_{2}^{0}\right| \\
& +\sum_{n \geq 1}\left|e_{1}^{n-1}\right\rangle\left\langle r_{+}^{\prime} e_{1}^{n}+s_{+}^{\prime} e_{2}^{n}|+| e_{2}^{n+1}\right\rangle\left\langle-e^{\mathrm{i} \mu_{3}^{\prime}} s_{+}^{\prime} e_{1}^{n}+e^{\mathrm{i} \mu_{3}^{\prime}} r_{+}^{\prime} e_{2}^{n}\right| \\
& +\sum_{n \leq-1}\left|e_{1}^{n-1}\right\rangle\left\langle r_{-}^{\prime} e_{1}^{n}+s_{-}^{\prime} e_{2}^{n}|+| e_{2}^{n+1}\right\rangle\left\langle-s_{-}^{\prime} e_{1}^{n}+r_{-}^{\prime} e_{2}^{n}\right| .
\end{aligned}
$$


Considering $P_{n \pm 1} e^{\mathrm{i} \lambda} W U_{r, \mu} W^{*} P_{n}=P_{n \pm 1} U_{r^{\prime}, \mu^{\prime}} P_{n}$ for any $n \in \mathbb{Z}$, we have $W e_{1}^{n}=$ $e^{\mathrm{i} u_{n}} e_{1}^{n}$ and $W e_{2}^{n}=e^{\mathrm{i} v_{n}} e_{2}^{n}$ for some $u_{n}, v_{n} \in \mathbb{R}$. Then,

$$
\begin{aligned}
e^{\mathrm{i} \lambda} & W U_{r, \mu} W^{*} \\
= & \left|e_{1}^{-1}\right\rangle\left\langle e^{\mathrm{i}\left(-u_{-1}+u_{0}-\lambda\right)} r_{0} e_{1}^{0}+e^{\mathrm{i}\left(-u_{-1}+v_{0}-\lambda+\mu_{1}\right)} s_{0} e_{2}^{0}\right| \\
& +\left|e_{2}^{1}\right\rangle\left\langle-e^{\mathrm{i}\left(-v_{1}+u_{0}-\lambda+\mu_{2}\right)} s_{0} e_{1}^{0}+e^{\mathrm{i}\left(-v_{1}+v_{0}-\lambda+\mu_{1}+\mu_{2}\right)} r_{0} e_{2}^{0}\right| \\
& +\sum_{n \geq 1}\left|e_{1}^{n-1}\right\rangle\left\langle e^{\mathrm{i}\left(-u_{n-1}+u_{n}-\lambda\right)} r_{+} e_{1}^{n}+e^{\mathrm{i}\left(-u_{n-1}+v_{n}-\lambda\right)} s_{+} e_{2}^{n}\right| \\
& +\left|e_{2}^{n+1}\right\rangle\left\langle-e^{\mathrm{i}\left(-v_{n+1}+u_{n}-\lambda+\mu_{3}\right)} s_{+} e_{1}^{n}+e^{\mathrm{i}\left(-v_{n+1}+v_{n}-\lambda+\mu_{3}\right)} r_{+} e_{2}^{n}\right| \\
& +\sum_{n \leq-1}\left|e_{1}^{n-1}\right\rangle\left\langle e^{\mathrm{i}\left(-u_{n-1}+u_{n}-\lambda\right)} r_{-} e_{1}^{n}+e^{\mathrm{i}\left(-u_{n-1}+v_{n}-\lambda\right)} s_{-} e_{2}^{n}\right| \\
& +\left|e_{2}^{n+1}\right\rangle\left\langle-e^{\mathrm{i}\left(-v_{n+1}+u_{n}-\lambda\right)} s_{-} e_{1}^{n}+e^{\mathrm{i}\left(-v_{n+1}+v_{n}-\lambda\right)} r_{-} e_{2}^{n}\right| .
\end{aligned}
$$

Since $e^{\mathrm{i} \lambda} W U_{r, \mu} W^{*}=U_{r^{\prime}, \mu^{\prime}}$, we obtain $r=r^{\prime}$. Moreover, comparing the coefficients of $\left|e_{1}^{n-1}\right\rangle\left\langle e_{1}^{n}|,| e_{1}^{n-1}\right\rangle\left\langle e_{2}^{n}\right|$ and $\left|e_{2}^{n+1}\right\rangle\left\langle e_{2}^{n}\right|$ yields

$$
-u_{n-1}+u_{n}-\lambda=0, \quad-u_{n-1}+v_{n}-\lambda=0(n \neq 0), \quad-v_{n+1}+v_{n}-\lambda=0(n \leq-1) .
$$

Here, we can assume that $u_{0}=0$, because $W U W^{*}=\left(e^{\mathrm{i} w} W\right) U\left(e^{\mathrm{i} w} W\right)^{*}$ for any $w \in \mathbb{R}$. Therefore, $u_{n}=n \lambda$, and this implies $v_{n}=u_{n-1}+\lambda=n \lambda(n \neq 0)$. Using the third equation in (10), we have $2 \lambda=0$, and therefore $\lambda=0$ or $\pi$. Moreover, the coefficients of $\left|e_{2}^{0}\right\rangle\left\langle e_{1}^{-1}\right|$ imply $v_{0}=u_{-1}-\lambda=-2 \lambda=0$. Comparing the coefficients of $\left|e_{1}^{-1}\right\rangle\left\langle e_{2}^{0}|,| e_{2}^{1}\right\rangle\left\langle e_{1}^{0}\right|$ and $\left|e_{2}^{n+1}\right\rangle\left\langle e_{2}^{n}\right|(n \geq 1)$, we obtain $\mu=\mu^{\prime}$.

This theorem says that the unitary equivalence classes of two-phase quantum walks with one defect are described by $\left\{U_{r, \mu}\right\}$ and are parameterized by six parameters.

From the above proof, if $e^{\mathrm{i} \lambda} W U_{r, \mu} W^{*}=U_{r, \mu}$, then $\lambda=0$ or $\pi$. When $\lambda=0$, $v_{n}=u_{n}=0$ and $W=I_{\mathcal{H}}$. When $\lambda=\pi, v_{n}=u_{n}=n \pi$ and $W=\bigoplus_{n \in \mathbb{Z}}(-1)^{n} I_{\mathcal{H}_{n}}$. Hence, we have the next corollary which we will use in Sect. 3.

Corollary 1 Let $0<r_{\varepsilon}<1(\varepsilon=+,-, 0)$ and $\mu_{i} \in[0,2 \pi)$, and let $W=\bigoplus_{n \in \mathbb{Z}} W_{n}$ be a unitary on $\mathcal{H}=\bigoplus_{n \in \mathbb{Z}} \mathcal{H}_{n}$. Then, for $\lambda \in[0,2 \pi)$,

$$
e^{\mathrm{i} \lambda} W U_{r, \mu} W^{*}=U_{r, \mu}
$$

if and only if $\lambda=0$ and $W=I_{\mathcal{H}}$ or $\lambda=\pi$ and $W=\bigoplus_{n \in \mathbb{Z}}(-1)^{n} I_{\mathcal{H}_{n}}$.

As a corollary of Theorem 3, we obtain the following.

Corollary 2 [12] A translation-invariant quantum walk $U$ is unitarily equivalent to

$$
U_{r}=\sum_{n \in \mathbb{Z}}\left|e_{1}^{n-1}\right\rangle\left\langle r e_{1}^{n}+s e_{2}^{n}|+| e_{2}^{n+1}\right\rangle\left\langle s e_{1}^{n}+r e_{2}^{n}\right|
$$

for some $0 \leq r \leq 1$ with $s=\sqrt{1-r^{2}}$. Moreover, $U_{r}$ and $U_{r^{\prime}}$ are unitarily equivalent if and only if $r=r^{\prime}$. 
Proof From the definition of a translation-invariant quantum walk, we can assume that, in (8), $r_{+}=r_{-}=r_{0}, a_{+}=a_{-}=a_{0}$ and so on. This implies that $\mu_{1}=b_{0}-b_{-}=0$, $\mu_{2}=b_{+}-b_{-}+c_{0}-c_{-}=0$ and $\mu_{3}=b_{+}-b_{-}+c_{+}-c_{-}=0$. Setting $r=r_{0}$ satisfies the first assertion. The necessary and sufficient condition for unitary equivalence follows from Theorem 3.

Corollary 3 A one-dimensional quantum walk $U$ with one defect is unitarily equivalent to

$$
\begin{aligned}
U_{r_{ \pm}, r_{0}, \nu_{1}, \nu_{2}}= & \left|e_{1}^{-1}\right\rangle\left\langle r_{0} e_{1}^{0}+e^{\mathrm{i} v_{1}} s_{0} e_{2}^{0}|+| e_{2}^{1}\right\rangle\left\langle-e^{\mathrm{i} \nu_{2}} s_{0} e_{1}^{0}+e^{\mathrm{i}\left(v_{1}+v_{2}\right)} r_{0} e_{2}^{0}\right| \\
& +\sum_{n \in \mathbb{Z} \backslash\{0\}}\left|e_{1}^{n-1}\right\rangle\left\langle r_{ \pm} e_{1}^{n}+s_{ \pm} e_{2}^{n}|+| e_{2}^{n+1}\right\rangle\left\langle s_{ \pm} e_{1}^{n}+r_{ \pm} e_{2}^{n}\right|
\end{aligned}
$$

for some $0 \leq r_{\varepsilon} \leq 1$ and $v_{1}, v_{2} \in \mathbb{R}$ with $s_{\varepsilon}=\sqrt{1-r_{\varepsilon}^{2}}(\varepsilon= \pm, 0)$. We write $U_{r_{ \pm}, r_{0}, v_{1}, v_{2}}=U_{r, v}$ for short. Moreover, when $0<r_{\varepsilon}, r_{\varepsilon}^{\prime}<1$ and $v_{i}, v_{i}^{\prime} \in[0,2 \pi)$, $U_{r, v}$ and $U_{r^{\prime}, v^{\prime}}$ are unitarily equivalent if and only if $r=r^{\prime}$ and $v=v^{\prime}$.

Proof From the definition of a one-dimensional quantum walk with one defect, we can assume that, in (8), $r_{+}=r_{-}, a_{+}=a_{-}$and so on. This implies that $\mu_{3}=$ $b_{+}-b_{-}+c_{+}-c_{-}=0$. Setting $r_{ \pm}=r_{+}, \nu_{1}=\mu_{1}$ and $\nu_{2}=\mu_{2}$ satisfies the first assertion. The necessary and sufficient condition for unitary equivalence follows from Theorem 3.

These corollaries imply that translation-invariant quantum walks are parameterized by one parameter and one-dimensional quantum walks with one defect by four parameters.

Clearly, Theorem 3 can be applied to complete two-phase quantum walks, though in this case, $U_{r, \mu}$ is not a complete two-phase quantum walk. Indeed, from the definition of complete two-phase quantum walks, we can assume that, in (8), $r_{0}=r_{+}, a_{0}=a_{+}$ and so on. Then, $\mu_{1}=b_{0}-b_{-} \neq 0$ in general, and the coefficients of $\left|e_{1}^{-1}\right\rangle\left\langle e_{2}^{0}\right|$ and $\left|e_{1}^{n-1}\right\rangle\left\langle e_{2}^{n}\right|(n \geq 1)$ of $U_{r, \mu}$ are not the same.

Hence, we provide the next theorem.

Theorem 4 A complete two-phase quantum walk $U$ is unitarily equivalent to

$$
\begin{aligned}
U_{r_{+}, r_{-}, \sigma_{1}, \sigma_{2}}= & \sum_{n \geq 0}\left|e_{1}^{n-1}\right\rangle\left\langle r_{+} e_{1}^{n}+s_{+} e_{2}^{n}|+| e_{2}^{n+1}\right\rangle\left\langle-e^{\mathrm{i} \sigma_{1}} s_{+} e_{1}^{n}+e^{\mathrm{i} \sigma_{1}} r_{+} e_{2}^{n}\right| \\
& +\sum_{n \leq-1}\left|e_{1}^{n-1}\right\rangle\left\langle r_{-} e_{1}^{n}+e^{\mathrm{i} \sigma_{2}} s_{-} e_{2}^{n}|+| e_{2}^{n+1}\right\rangle\left\langle-s_{-} e_{1}^{n}+e^{\mathrm{i} \sigma_{2}} r_{-} e_{2}^{n}\right|
\end{aligned}
$$

for some $0 \leq r_{+}, r_{-} \leq 1$ and $\sigma_{1}, \sigma_{2} \in \mathbb{R}$ with $s_{\varepsilon}=\sqrt{1-r_{\varepsilon}^{2}}(\varepsilon=+,-)$. We write $U_{r_{+}, r_{-}, \sigma_{1}, \sigma_{2}}=U_{r, \sigma}$ for short. Moreover, when $0<r_{\varepsilon}, r_{\varepsilon}^{\prime}<1$ and $\sigma_{i}, \sigma_{i}^{\prime} \in[0,2 \pi)$, $U_{r, \sigma}$ and $U_{r^{\prime}, \sigma^{\prime}}$ are unitarily equivalent if and only if $r=r^{\prime}$ and $\sigma=\sigma^{\prime}$. 
Proof The proof is almost the same as that given for Theorem 3, but we present it here for completeness. Let

$$
U=\sum_{n \in \mathbb{Z}}\left|\xi_{n-1, n}\right\rangle\left\langle\zeta_{n-1, n}|+| \xi_{n+1, n}\right\rangle\left\langle\zeta_{n+1, n}\right|
$$

be a complete two-phase quantum walk. Then, by definition, Eq. (2) can be written as

$$
\begin{aligned}
W_{1} U W_{1}^{*}= & \sum_{n \geq 0}\left|e_{1}^{n-1}\right\rangle\left\langle e^{\mathrm{i} a_{+}} r_{+} e_{1}^{n}+e^{\mathrm{i} b_{+}} s_{+} e_{2}^{n}|+| e_{2}^{n+1}\right\rangle\left\langle e^{\mathrm{i} c_{+}} s_{+} e_{1}^{n}+e^{\mathrm{i} d_{+}} r_{+} e_{2}^{n}\right| \\
& +\sum_{n \leq-1}\left|e_{1}^{n-1}\right\rangle\left\langle e^{\mathrm{i} a_{-}} r_{-} e_{1}^{n}+e^{\mathrm{i} b_{-}} s_{-} e_{2}^{n}|+| e_{2}^{n+1}\right\rangle\left\langle e^{\mathrm{i} c_{-}} s_{-} e_{1}^{n}+e^{\mathrm{i} d_{-}} r_{-} e_{2}^{n}\right|
\end{aligned}
$$

for some $0 \leq r_{\varepsilon} \leq 1$ and $a_{\varepsilon}, b_{\varepsilon}, c_{\varepsilon}, d_{\varepsilon} \in \mathbb{R}$ with $s_{\varepsilon}=\sqrt{1-r_{\varepsilon}^{2}}(\varepsilon=+,-)$.

We then modify Step 2 as follows:

Step $2^{\prime \prime}$ Let $\ell=\left(b_{+}+c_{-}+\pi\right) / 2$. Define $g_{n}, h_{n} \in \mathbb{R}$ by

$$
g_{n}= \begin{cases}n\left(\ell-a_{+}\right) & (n \geq 0) \\ n\left(\ell-a_{-}\right)-a_{-}+a_{+} & (n \leq-1)\end{cases}
$$

and

$$
h_{n}=\left\{\begin{array}{ll}
(n-1)\left(\ell-a_{+}\right)-b_{+}+\ell & (n \geq 1) \\
(n-1)\left(\ell-a_{-}\right)+c_{-}+a_{+}-a_{-}-\ell+\pi & (n \leq 0)
\end{array},\right.
$$

and a unitary $W_{2}$ on $\mathcal{H}$ by

$$
W_{2}=\bigoplus_{n \in \mathbb{Z}} e^{\mathrm{i} g_{n}}\left|e_{1}^{n}\right\rangle\left\langle e_{1}^{n}\left|+e^{\mathrm{i} h_{n}}\right| e_{2}^{n}\right\rangle\left\langle e_{2}^{n}\right| .
$$

Then, using $a_{\varepsilon}+d_{\varepsilon}+\pi=b_{\varepsilon}+c_{\varepsilon}$,

$$
\begin{aligned}
e^{\mathrm{i} \ell} & W_{2} W_{1} U W_{1}^{*} W_{2}^{*} \\
= & \sum_{n \geq 0}\left|e_{1}^{n-1}\right\rangle\left\langle e^{\mathrm{i}\left(a_{+}-g_{n-1}+g_{n}-\ell\right)} r_{+} e_{1}^{n}+e^{\mathrm{i}\left(b_{+}-g_{n-1}+h_{n}-\ell\right)} s_{+} e_{2}^{n}\right| \\
& +\left|e_{2}^{n+1}\right\rangle\left\langle e^{\mathrm{i}\left(c_{+}-h_{n+1}+g_{n}-\ell\right)} s_{+} e_{1}^{n}+e^{\mathrm{i}\left(d_{+}-h_{n+1}+h_{n}-\ell\right)} r_{+} e_{2}^{n}\right| \\
& +\sum_{n \leq-1}\left|e_{1}^{n-1}\right\rangle\left\langle e^{\mathrm{i}\left(a_{-}-g_{n-1}+g_{n}-\ell\right)} r_{-} e_{1}^{n}+e^{\mathrm{i}\left(b_{-}-g_{n-1}+h_{n}-\ell\right)} s_{-} e_{2}^{n}\right| \\
& +\left|e_{2}^{n+1}\right\rangle\left\langle e^{\mathrm{i}\left(c_{-}-h_{n+1}+g_{n}-\ell\right)} s_{-} e_{1}^{n}+e^{\mathrm{i}\left(d_{-}-h_{n+1}+h_{n}-\ell\right)} r_{-} e_{2}^{n}\right| \\
= & \sum_{n \geq 0}\left|e_{1}^{n-1}\right\rangle\left\langle r_{+} e_{1}^{n}+s_{+} e_{2}^{n}|+| e_{2}^{n+1}\right\rangle\left\langle-e^{\mathrm{i}\left(c_{+}-c_{-}\right)} s_{+} e_{1}^{n}+e^{\mathrm{i}\left(c_{+}-c_{-}\right)} r_{+} e_{2}^{n}\right| \\
& +\sum_{n \leq-1}\left|e_{1}^{n-1}\right\rangle\left\langle r_{-} e_{1}^{n}+e^{\mathrm{i}\left(b_{-}-b_{+}\right)} s_{-} e_{2}^{n}|+| e_{2}^{n+1}\right\rangle\left\langle-s_{-} e_{1}^{n}+e^{\mathrm{i}\left(b_{-}-b_{+}\right)} r_{-} e_{2}^{n}\right|
\end{aligned}
$$




$$
\begin{aligned}
= & \sum_{n \geq 0}\left|e_{1}^{n-1}\right\rangle\left\langle r_{+} e_{1}^{n}+s_{+} e_{2}^{n}|+| e_{2}^{n+1}\right\rangle\left\langle-e^{\mathrm{i} \sigma_{1}} s_{+} e_{1}^{n}+e^{\mathrm{i} \sigma_{1}} r_{+} e_{2}^{n}\right| \\
& +\sum_{n \leq-1}\left|e_{1}^{n-1}\right\rangle\left\langle r_{-} e_{1}^{n}+e^{\mathrm{i} \sigma_{2}} s_{-} e_{2}^{n}|+| e_{2}^{n+1}\right\rangle\left\langle-s_{-} e_{1}^{n}+e^{\mathrm{i} \sigma_{2}} r_{-} e_{2}^{n}\right| \\
= & U_{r, \sigma},
\end{aligned}
$$

where $\sigma_{1}=c_{+}-c_{-}$and $\sigma_{2}=b_{-}-b_{+}$. This shows the first assertion of this theorem.

Next, assume that $U_{r, \sigma}$ and $U_{r^{\prime}, \sigma^{\prime}}$ are unitarily equivalent, where $0<r_{\varepsilon}, r_{\varepsilon}^{\prime}<1$ and $\sigma_{i}, \sigma_{i}^{\prime} \in[0,2 \pi)$. Then, there exist $\lambda \in \mathbb{R}$ and a unitary operator $W=\bigoplus_{n \in \mathbb{Z}} W_{n}$ on $\mathcal{H}=\bigoplus_{n \in \mathbb{Z}} \mathcal{H}_{n}$ such that

$$
e^{\mathrm{i} \lambda} W U_{r, \sigma} W^{*}=U_{r^{\prime}, \sigma^{\prime}}
$$

Here,

$$
\begin{aligned}
& e^{\mathrm{i} \lambda} W U_{r, \sigma} W^{*} \\
& =e^{\mathrm{i} \lambda} \sum_{n \geq 0}\left|W e_{1}^{n-1}\right\rangle\left\langle r_{+} W e_{1}^{n}+s_{+} W e_{2}^{n}|+| W e_{2}^{n+1}\right\rangle\left\langle-e^{\mathrm{i} \sigma_{1}} s_{+} W e_{1}^{n}+e^{\mathrm{i} \sigma_{1}} r_{+} W e_{2}^{n}\right| \\
& \quad+\sum_{n \leq-1}\left|W e_{1}^{n-1}\right\rangle\left\langle r_{-} W e_{1}^{n}+e^{\mathrm{i} \sigma_{2}} s_{-} W e_{2}^{n}|+| W e_{2}^{n+1}\right\rangle\left\langle-s_{-} W e_{1}^{n}+e^{\mathrm{i} \sigma_{2}} r_{-} W e_{2}^{n}\right|
\end{aligned}
$$

and

$$
\begin{aligned}
U_{r^{\prime}, \sigma^{\prime}}= & \sum_{n \geq 0}\left|e_{1}^{n-1}\right\rangle\left\langle r_{+}^{\prime} e_{1}^{n}+s_{+}^{\prime} e_{2}^{n}|+| e_{2}^{n+1}\right\rangle\left\langle-e^{\mathrm{i} \sigma_{1}^{\prime}} s_{+}^{\prime} e_{1}^{n}+e^{\mathrm{i} \sigma_{1}^{\prime}} r_{+}^{\prime} e_{2}^{n}\right| \\
& +\sum_{n \leq-1}\left|e_{1}^{n-1}\right\rangle\left\langle r_{-}^{\prime} e_{1}^{n}+e^{\mathrm{i} \sigma_{2}^{\prime}} s_{-}^{\prime} e_{2}^{n}|+| e_{2}^{n+1}\right\rangle\left\langle-s_{-}^{\prime} e_{1}^{n}+e^{\mathrm{i} \sigma_{2}^{\prime}} r_{-}^{\prime} e_{2}^{n}\right| .
\end{aligned}
$$

Considering $P_{n \pm 1} e^{\mathrm{i} \lambda} W U_{r, \sigma} W^{*} P_{n}=P_{n \pm 1} U_{r^{\prime}, \sigma^{\prime}} P_{n}$ for any $n \in \mathbb{Z}$, we have $W e_{1}^{n}=$ $e^{\mathrm{i} u_{n}} e_{1}^{n}$ and $W e_{2}^{n}=e^{\mathrm{i} v_{n}} e_{2}^{n}$ for some $u_{n}, v_{n} \in \mathbb{R}$. Then,

$$
\begin{aligned}
& e^{\mathrm{i} \lambda} W U_{r, \sigma} W^{*} \\
& =\sum_{n \geq 0}\left|e_{1}^{n-1}\right\rangle\left\langle e^{\mathrm{i}\left(-u_{n-1}+u_{n}-\lambda\right)} r_{+} e_{1}^{n}+e^{\mathrm{i}\left(-u_{n-1}+v_{n}-\lambda\right)} s_{+} e_{2}^{n}\right| \\
& \quad+\left|e_{2}^{n+1}\right\rangle\left\langle-e^{\mathrm{i}\left(-v_{n+1}+u_{n}-\lambda+\sigma_{1}\right)} s_{+} e_{1}^{n}+e^{\mathrm{i}\left(-v_{n+1}+v_{n}-\lambda+\sigma_{1}\right)} r_{+} e_{2}^{n}\right| \\
& \quad+\sum_{n \leq-1}\left|e_{1}^{n-1}\right\rangle\left\langle e^{\mathrm{i}\left(-u_{n-1}+u_{n}-\lambda\right)} r_{-} e_{1}^{n}+e^{\mathrm{i}\left(-u_{n-1}+v_{n}-\lambda+\sigma_{2}\right)} s_{-} e_{2}^{n}\right| \\
& \quad+\left|e_{2}^{n+1}\right\rangle\left\langle-e^{\mathrm{i}\left(-v_{n+1}+u_{n}-\lambda\right)} s_{-} e_{1}^{n}+e^{\mathrm{i}\left(-v_{n+1}+v_{n}-\lambda+\sigma_{2}\right)} r_{-} e_{2}^{n}\right| .
\end{aligned}
$$

Since $e^{\mathrm{i} \lambda} W U_{r, \sigma} W^{*}=U_{r^{\prime}, \sigma^{\prime}}$, we obtain $r=r^{\prime}$. Moreover, comparing the coefficients of $\left|e_{1}^{n-1}\right\rangle\left\langle e_{1}^{n}|,| e_{1}^{n-1}\right\rangle\left\langle e_{2}^{n}\right|$ and $\left|e_{2}^{n+1}\right\rangle\left\langle e_{1}^{n}\right|$ yields

$-u_{n-1}+u_{n}-\lambda=0, \quad-u_{n-1}+v_{n}-\lambda=0(n \geq 0), \quad-v_{n+1}+u_{n}-\lambda=0(n \leq-1)$. 
Here, we can assume that $u_{0}=0$, because $W U W^{*}=\left(e^{\mathrm{i} w} W\right) U\left(e^{\mathrm{i} w} W\right)^{*}$ for any $w \in \mathbb{R}$. Therefore, $u_{n}=n \lambda$, and this implies $v_{n}=u_{n-1}+\lambda=n \lambda(n \geq 0)$ and $v_{n+1}=u_{n}-\lambda=(n-1) \lambda(n \leq-1)$. Hence, $v_{0}=0$ and $v_{0}=-2 \lambda$, with the result that $\lambda=0$ or $\pi$. Then, $-v_{n+1}+v_{n}-\lambda=0$ for all $n \in \mathbb{Z}$, and therefore, $\sigma=\sigma^{\prime}$, comparing the coefficients of $\left|e_{2}^{n+1}\right\rangle\left\langle e_{2}^{n}\right|$. This completes the proof.

This theorem says that complete two-phase quantum walks are parameterized by four parameters.

From the above proof, if $e^{\mathrm{i} \lambda} W U_{r, \sigma} W^{*}=U_{r, \sigma}$, then $\lambda=0$ or $\pi$. When $\lambda=0$, $v_{n}=u_{n}=0$ and $W=I_{\mathcal{H}}$. When $\lambda=\pi, v_{n}=u_{n}=n \pi$ and $W=\bigoplus_{n \in \mathbb{Z}}(-1)^{n} I_{\mathcal{H}_{n}}$. Hence, we have the next corollary.

Corollary 4 Let $0<r_{\varepsilon}<1(\varepsilon=+,-)$ and $\sigma_{i} \in[0,2 \pi)(i=1,2)$, and let $W=\bigoplus_{n \in \mathbb{Z}} W_{n}$ be a unitary on $\mathcal{H}=\bigoplus_{n \in \mathbb{Z}} \mathcal{H}_{n}$. Then, for $\lambda \in[0,2 \pi)$,

$$
e^{\mathrm{i} \lambda} W U_{r, \sigma} W^{*}=U_{r, \sigma}
$$

if and only if $\lambda=0$ and $W=I_{\mathcal{H}}$ or $\lambda=\pi$ and $W=\bigoplus_{n \in \mathbb{Z}}(-1)^{n} I_{\mathcal{H}_{n}}$.

\section{Unitary equivalence classes of one-dimensional quantum walks with initial states}

In this section, we consider one-dimensional quantum walks with initial states. We assume that an initial state $\Phi$ is in $\mathcal{H}_{0}$.

Definition 4 One-dimensional quantum walks with initial states $(U, \Phi)$ and $\left(U^{\prime}, \Phi^{\prime}\right)$ are unitarily equivalent if there exists a unitary $W=\bigoplus_{n \in \mathbb{Z}} W_{n}$ on $\mathcal{H}=\bigoplus_{n \in \mathbb{Z}} \mathcal{H}_{n}$ such that

$$
U^{\prime}=W U W^{*} \text { and } \Phi^{\prime}=W \Phi .
$$

Unitary equivalence classes of two-phase quantum walks with one defect with initial states are described as follows:

Theorem 5 A two-phase quantum walk with one defect with an initial state $(U, \Phi)$ is unitarily equivalent to $\left(U_{r, \mu}, \Phi_{\alpha, \theta}\right)$ for some $0 \leq r_{\varepsilon}, \alpha \leq 1(\varepsilon=+,-, 0), \mu_{i}, \theta \in \mathbb{R}$ $(i=1,2,3)$, where $\Phi_{\alpha, \theta}=\alpha e_{1}^{0}+e^{\mathrm{i} \theta} \sqrt{1-\alpha^{2}} e_{2}^{0}$.

Moreover, $\left(U_{r, \mu}, \Phi_{\alpha, \theta}\right)$ and $\left(U_{r^{\prime}, \mu^{\prime}}, \Phi_{\alpha^{\prime}, \theta^{\prime}}\right)$ with $0<r_{\varepsilon}, r_{\varepsilon}^{\prime}, \alpha, \alpha^{\prime}<1$ and $\mu_{i}, \mu_{i}^{\prime}, \theta, \theta^{\prime} \in[0,2 \pi)$ are unitarily equivalent if and only if $r=r^{\prime}, \mu=\mu^{\prime}, \alpha=\alpha^{\prime}$ and $\theta=\theta^{\prime}$.

Proof It was proved that $U$ is unitarily equivalent to $U_{r, \mu}$ for some $r$ and $\mu$ in Theorem 3. Hence, there exists a unitary $W=\bigoplus_{n \in \mathbb{Z}} W_{n}$ on $\mathcal{H}$ such that $W U W^{*}=U_{r, \mu}$. The state $W \Phi \in \mathcal{H}_{0}=\mathbb{C}^{2}$ can be written as $W \Phi=\alpha e_{1}^{0}+\beta e_{2}^{0}$ for some $\alpha, \beta \in \mathbb{C}$ with $|\alpha|^{2}+|\beta|^{2}=1$. Since $W \Phi$ and $e^{\mathrm{i} \lambda} W \Phi$ are identified, we can assume that $0 \leq \alpha \leq 1$. Then, $\beta=e^{\mathrm{i} \theta} \sqrt{1-\alpha^{2}}$ for some $\theta \in \mathbb{R}$. Therefore, $W \Phi=\Phi_{\alpha, \theta}$ holds, and hence, $(U, \Phi)$ is unitarily equivalent to $\left(U_{r, \mu}, \Phi_{\alpha, \theta}\right)$. 
Next, assume that $\left(U_{r, \mu}, \Phi_{\alpha, \theta}\right)$ and $\left(U_{r^{\prime}, \mu^{\prime}}, \Phi_{\alpha^{\prime}, \theta^{\prime}}\right)$ with $0<r_{\varepsilon}, r_{\varepsilon}^{\prime}, \alpha, \alpha^{\prime}<1$ and $\mu_{i}, \mu_{i}^{\prime}, \theta, \theta^{\prime} \in[0,2 \pi)$ are unitarily equivalent. Then, by Theorem $3, r=r^{\prime}$ and $\mu=\mu^{\prime}$. Moreover, if there exist $\lambda \in \mathbb{R}$ and a unitary operator $W=\bigoplus_{n \in \mathbb{Z}} W_{n}$ on $\mathcal{H}$ such that

$$
e^{\mathrm{i} \lambda} W U_{r, \mu} W^{*}=U_{r, \mu}
$$

then, by Corollary $1, \lambda=0$ and $W=I$ or $\lambda=\pi$ and $W=\bigoplus_{n \in \mathbb{Z}}(-1)^{n} I_{\mathcal{H}_{n}}$. Therefore, $W \Phi_{\alpha, \theta}=\Phi_{\alpha^{\prime}, \theta^{\prime}}$ implies $\alpha=\alpha^{\prime}$ and $\theta=\theta^{\prime}$.

As corollaries, and from Corollaries 2 and 3, we have the following.

Corollary 5 A translation-invariant quantum walk $(U, \Phi)$ is unitarily equivalent to $\left(U_{r}, \Phi_{\alpha, \theta}\right)$ for some $0 \leq r, \alpha \leq 1$ and $\theta \in \mathbb{R}$.

Moreover, $\left(U_{r}, \Phi_{\alpha, \theta}\right)$ and $\left(\bar{U}_{r^{\prime}}, \Phi_{\alpha^{\prime}, \theta^{\prime}}\right)$ with $0<r, r^{\prime}, \alpha, \alpha^{\prime}<1$ and $\theta, \theta^{\prime} \in[0,2 \pi)$ are unitarily equivalent if and only if $r=r^{\prime}, \alpha=\alpha^{\prime}$ and $\theta=\theta^{\prime}$.

Corollary 6 A one-dimensional quantum walk with one defect $(U, \Phi)$ is unitarily equivalent to $\left(U_{r, v}, \Phi_{\alpha, \theta}\right)$ for some $0 \leq r_{\varepsilon}, \alpha \leq 1(\varepsilon= \pm, 0)$ and $v_{i}, \theta \in \mathbb{R}(i=1,2)$.

Moreover, $\left(U_{r, v}, \Phi_{\alpha, \theta}\right)$ and $\left(U_{r^{\prime}, v^{\prime}}, \Phi_{\alpha^{\prime}, \theta^{\prime}}\right)$ with $0<r_{\varepsilon}, r_{\varepsilon}^{\prime}, \alpha, \alpha^{\prime}<1$ and $v_{i}, v_{i}^{\prime}, \theta, \theta^{\prime} \in[0,2 \pi)$ are unitarily equivalent if and only if $r=r^{\prime}, v=v^{\prime}, \alpha=\alpha^{\prime}$ and $\theta=\theta^{\prime}$.

The proof of the next theorem is almost the same as that given for Theorem 5 and is omitted.

Theorem 6 A complete two-phase quantum walk $(U, \Phi)$ is unitarily equivalent to $\left(U_{r, \sigma}, \Phi_{\alpha, \theta}\right)$ for some $0 \leq r_{\varepsilon}, \alpha \leq 1(\varepsilon=+,-)$ and $\sigma_{i}, \theta \in \mathbb{R}(i=1,2)$.

Moreover, $\left(U_{r, \sigma}, \Phi_{\alpha, \theta}\right)$ and $\left(U_{r^{\prime}, \sigma^{\prime}}, \Phi_{\alpha^{\prime}, \theta^{\prime}}\right)$ with $0<r_{\varepsilon}, r_{\varepsilon}^{\prime}, \alpha, \alpha^{\prime}<1$ and $\sigma_{i}, \sigma_{i}^{\prime}, \theta, \theta^{\prime} \in[0,2 \pi)$ are unitarily equivalent if and only if $r=r^{\prime}, \sigma=\sigma^{\prime}, \alpha=\alpha^{\prime}$ and $\theta=\theta^{\prime}$.

\section{Conclusion}

We discussed the unitary equivalence classes of some types of one-dimensional quantum walks with or without initial states. In the previous paper [19], we showed some general properties of unitarily equivalent quantum walks. In particular, we proved that every one-dimensional quantum walk is the unitarily equivalent of one of the Ambainis types. We also presented the necessary and sufficient condition for defining a one-dimensional quantum walk as a Szegedy walk. In the present paper, we studied the unitary equivalence classes of some special types of one-dimensional quantum walks. As a consequence, we obtained the following results: Two-phase quantum walks with one defect (without initial states) are parameterized by six parameters, complete two-phase quantum walks by four parameters and one-dimensional quantum walks with one defect by four parameters. When we consider quantum walks with initial states, we need additional two parameters which correspond to the initial state $\Phi_{\alpha, \theta}$. 
Moreover, we showed that the unitary equivalence classes of general one-dimensional quantum walks are described by $\left\{U_{r, \theta}\right\}$ with $\theta_{0}=\theta_{1}=0$.

The parameterization of unitary equivalence classes tells us what part of onedimensional quantum walks were already investigated and what part should be studied. For example, a complete two-phase quantum walk considered in [11] is defined by

$$
\begin{aligned}
U^{(s)}= & \frac{1}{\sqrt{2}} \sum_{n \geq 0}\left|e_{1}^{n-1}\right\rangle\left\langle e_{1}^{n}+e^{\mathrm{i} \sigma_{+}} e_{2}^{n}|+| e_{2}^{n+1}\right\rangle\left\langle e^{-\mathrm{i} \sigma_{+}} e_{1}^{n}-e_{2}^{n}\right| \\
& +\frac{1}{\sqrt{2}} \sum_{n \leq-1}\left|e_{1}^{n-1}\right\rangle\left\langle e_{1}^{n}+e^{\mathrm{i} \sigma_{-}} e_{2}^{n}|+| e_{2}^{n+1}\right\rangle\left\langle e^{-\mathrm{i} \sigma_{-}} e_{1}^{n}-e_{2}^{n}\right|
\end{aligned}
$$

for some $\sigma_{+}, \sigma_{-} \in[0,2 \pi)$. From Theorem $4, U^{(s)}$ is unitarily equivalent to $U_{r, \sigma}$ with $r_{+}=r_{-}=1 / \sqrt{2}$ and $\sigma_{1}=\sigma_{2}=\sigma_{-}-\sigma_{+}$. Hence, quantum walks $U_{r, \sigma}$ with $\sigma_{1} \neq \sigma_{2}$ are not included in the model of $U^{(s)}$. Furthermore, we can reduce the parameters defining $\sigma_{1}=\sigma_{2}=\sigma_{-}-\sigma_{+}$. In the same way, we can apply our results to many other previous works.

Acknowledgements The author is grateful to Etsuo Segawa and Akito Suzuki for their helpful comments and suggestions. This work was supported by JSPS KAKENHI Grant No. 17K05274.

Open Access This article is distributed under the terms of the Creative Commons Attribution 4.0 International License (http://creativecommons.org/licenses/by/4.0/), which permits unrestricted use, distribution, and reproduction in any medium, provided you give appropriate credit to the original author(s) and the source, provide a link to the Creative Commons license, and indicate if changes were made.

\section{References}

1. Aharanov, L., Davidovidh, N., Zagury, N.: Quantum random walks. Phys. Rev. A 48, 1687-1690 (1993)

2. Ambainis, A., Bach, E., Nayak, A., Vishwanath, A., Watrous, J.: One-dimensional quantum walks. In: Proceedings of 33th ACM Symposium of the Theory of Computing, pp. 37-49 (2001)

3. Cantero, M.J., Grünbaum, F.A., Moral, L., Velázquez, L.: Matrix valued Szegő polynomials and quantum random walks. Commun. Pure Appl. Math. 58, 464-507 (2010)

4. Cantero, M.J., Grünbaum, F.A., Moral, L., Velázquez, L.: One-dimensional quantum walks with one defect. Rev. Math. Phys. 24, 1250002 (2012)

5. Cantero, M.J., Grünbaum, F.A., Moral, L., Velázquez, L.: The CGMV method for quantum walks. Quantum Inf. Process. 11, 1149-1192 (2012)

6. Endo, S., Endo, T., Konno, N., Segawa, E., Takei, M.: Weak limit theorem of a two-phase quantum walk with one defect. arXiv:1412.4309

7. Endo, S., Endo, T., Konno, N., Segawa, E., Takei, M.: Limit theorems of a two-phase quantum walk with one defect. Quantum Inf. Comput. 15, 1373-1396 (2015)

8. Endo, S., Konno, N.: Weak convergence of the Wojcik model. Yokohama Math. J. 61, 87-111 (2015)

9. Endo, S., Konno, N.: The stationary measure of a space-inhomogeneous quantum walk on the line. Yokohama Math. J. 60, 33-47 (2014)

10. Endo, S., Konno, N., Segawa, E., Takei, M.: A one-dimensional Hadamard walk with one defect. Yokohama Math. J. 60, 49-90 (2014)

11. Endo, T., Konno, N., Obuse, H.: Relation between two-phase quantum walks and the topological invariant. arXiv:1511.04230

12. Goyal, S.K., Konrad, T., Diósi, L.: Unitary equivalence of quantum walks. Phys. Lett. A 379, 100-104 (2015) 
13. Gudder, S.P.: Quantum Probability. Academic Press, London (1988)

14. Kitagawa, T., Rudner, M.S., Berg, E., Demler, E.: Exploring topological phases with quantum walks. Phys. Rev. A 82, 033429 (2010)

15. Konno, N.: Quantum random walks in one dimensional. Quantum Inf. Process. 1, 345-354 (2002)

16. Konno, N.: One-dimensional discrete-time quantum walks on random environment. Quantum Inf. Process. 8, 387-399 (2009)

17. Konno, N.: Localization of an inhomogeneous discrete-time quantum walk on the line. Quantum Inf. Process. 9, 405-418 (2010)

18. Konno, N., Łuczak, T., Segawa, E.: Limit measures of inhomogeneous discrete-time quantum walks in one dimensional. Quantum Inf. Process. 12, 33-53 (2013)

19. Ohno, H.: Unitary equivalent classes of one-dimensional quantum walks. Quantum Inf. Process. 15, 3599-3617 (2016)

20. Segawa, E., Suzuki, A.: Generator of an abstract quantum walk. Quantum Stud. Math. Found. 3, 11-30 (2016)

21. Shikano, Y., Katsura, H.: Localization and fractality in inhomogeneous quantum walks with selfduality. Phys. Rev. E 82, 031122 (2010)

22. Venegas-Andraca, S.E.: Quantum walks: a comprehensive review. Quantum Inf. Process. 11, 10151106 (2012)

23. Wójcik, A., Łuczak, T., Kurzyński, P., Grudka, A., Gdala, T., Bednarska-Bzdega, M.: Trapping a particle of a quantum walk on the line. Phys. Rev. A 85, 012329 (2012) 